\title{
VORTEX SIPHON - FROM 1:1 SCALE PHYSICAL MODEL TO SPH SIMULATION AND PROTOTYPE
}

\author{
Arnaud Bart ${ }^{1}$, Thibault Macherel ${ }^{1}$, De Cesare Giovanni ${ }^{2}$, Sean Mulligan ${ }^{3}$, Khalid Essyad ${ }^{4}$ \\ ${ }^{1}$ Section of Mechanical Engineering, Faculté des Sciences et Techniques de l'Ingénieur, (STI), Ecole \\ Polytechnique Fédérale de Lausanne (EPFL), Lausanne, Switzerland, arnaud.bart@epfl.ch, \\ thibault.macherel@epfl.ch
}

${ }^{2}$ Plateforme de Constructions Hydrauliques, Faculté de l'Environnement Naturel, Architectural et Construit (ENAC), Ecole Polytechnique Fédérale de Lausanne (EPFL), Lausanne, Switzerland, giovanni.decesare@epfl.ch
${ }^{3}$ National University of Ireland, Galway, Department of Civil Engineering, College of Engineering and Informatics, Co. Galway, Ireland, sean.mulligan@nuigalway.ie

${ }^{4}$ BG Ingénieurs Conseils, Eau \& Environnement, Av. de Cour 61, CP 241, 1001 Lausanne, Switzerland, khalid.essyad@bg-21.com

Key words: Vortex, free surface, water transfer, groundwater, SPH modelling

\begin{abstract}
The rail link between Geneva Cornavin Station, Eaux-Vives Station and Annemasse (CEVA) is currently under construction including a cut-and-cover tunnel built by slurry walls located in the alluvium of the Foron River. The covered trench cuts the groundwater table flow which causes a rise of the water table on one side of the tunnel. Thirty wells were built in order to convey water from the aquifer on one side of the tunnel to the other. For each well, traditional pumping has been replaced by a self-priming multiphase siphon in order to lower energy costs. The operating principle of the system is based on the sustaining of a free-surface vortex flow in a vacuum chamber. In this way, the multiphase siphon is primed to allow water to be transferred from either side of the tunnel. A physical model of the conveyance system has been constructed at a scale of 1:1 in order to avoid scale effects from the presence of air water mixture and due to the free-surface vortices sensitivity to scaling, particularly in a vacuum atmosphere. In this study, the results from the physical model are used to validate a Smoothed Particle Hydrodynamics (SPH) numerical simulation of the complex flow conditions. The DualSPHysics code is employed and the results presented herein regarding the depth discharge relationship are encouraging for further application in vortex flows and such devices where conventional Eulerian modelling approaches can be challenging. As far as the authors are aware, this is the first application of SPH in simulating vortex chamber flows and the result bodes well for further application in this area.
\end{abstract}

\section{INTRODUCTION}

Three-dimensional numerical simulations are nowadays widely used in hydraulics and fluid dynamics [1]. It saves material, time and financial resources while at the same time, enables vast access to three-dimensional, time dependent data for various flow processes. Students at the EPFL (Ecole Polytechnique Fédérale de Lausanne) have the opportunity to learn how to simulate physical experiments with different methods and software. One course called "Particle-based Methods" [2] delivered by Professor Mark Sawley of the 
mechanical engineering section, gives in-depth training on how to simulate experiments using DEM (Discrete element method) or SPH (Smooth Particle Hydrodynamics) method. During this class in early 2018, two students have chosen to apply SPH numerical modelling to free-surface vortex flows to verify its accuracy and advantages in modelling such flow cases. The software "DualSPHysics" was employed and physical data from a 1:1 scaled physical model was used to validate the simulation results. The physical model used for validation is part of a study developed previously [3] for the CEVA project (Cornavin-Eaux-Vives-Annemasse) described next.

This new rail double track link between Geneva main Cornavin Station, Eaux-Vives Station and Annemasse (CEVA) [4] is currently under construction including a cut-and-cover tunnel built with slurry walls located in the alluvium of the Foron River. Near Eaux-Vives Train Station, the rail line crosses the Foron River. The impact of the tunnel on the hydrogeology was problematic where it acted as a cut-off for groundwater flow, leading to differing levels of groundwater on either side of the tunnel. In order to restore groundwater equilibrium, an innovative self-priming vortex siphon system was developed to transfer water over the tunnel (see Figure 1 and Figure 2) [3]. This system avoids the need for pumps and thus saves energy costs for the entire life of the tunnel.

\section{PUITS DE CAPTAGE ET SYSTEME DE SIPHON}

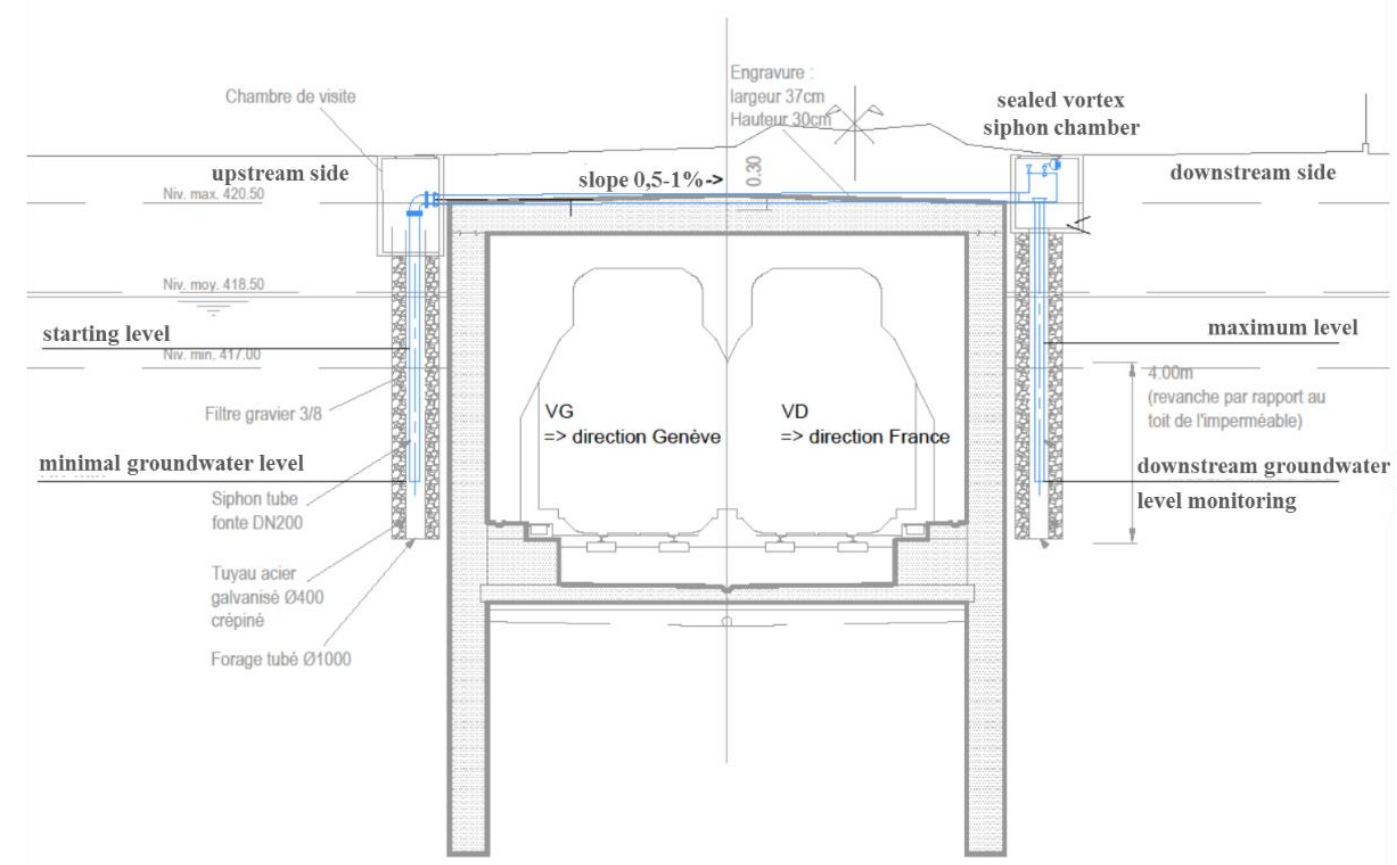

Figure 1: Cross section of the CEVA tunnel with water transfer system

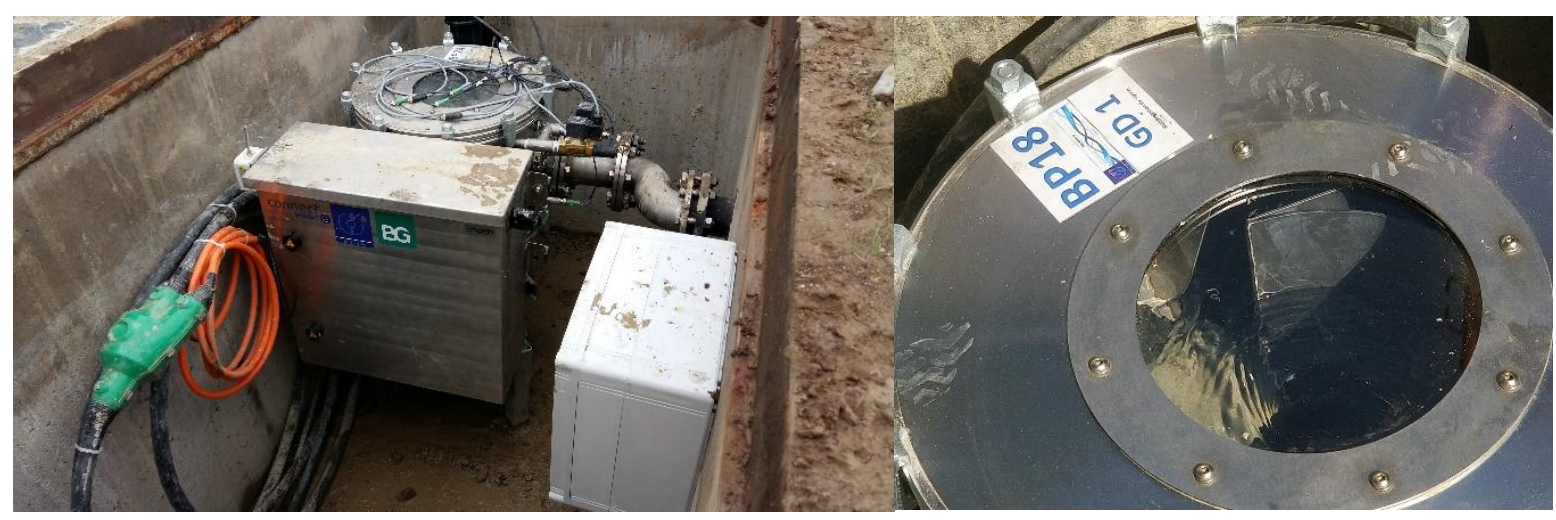

Figure 2: Installed prototype sealed vortex siphon (courtesy of BG) 
A vortex chamber is installed in the siphon circuit, where the multiphase conditions of the vortex flow permits air transport from leaks, damaged pipes and potentially dissolved air which would otherwise suppress the siphon if it was to perform as a single phase flow. The box containing the vortex chamber is sealed but can have its inner pressure varied using a suction pump and air vent. The performance of the siphon is largely dependent on the strength of the ability of the flow to entrain air and convey it down under various pressure conditions. The design of the vortex chamber thus underwent numerous physical iterations in the projects initial stages [3].

In order to avoid such costs in physical modelling in future studies, the purpose of this paper is to assess the validity of applying smoothed particle hydrodynamics (SPH) to vortex flows. Previous studies [5, 6] show that, although it is possible to simulate free-surface vortices to within a respectable degree of accuracy using Eulerian multiphase approaches, the time required during the meshing process, not to mention computational power $[5,6]$ to perform the simulations is discouraging. On the other hand, the DualSPHysics SPH code has been developed to study free-surface flow phenomena where Eulerian methods can be difficult to apply. It uses a set of $\mathrm{C}++$, CUDA and Java codes designed to deal with real-life engineering problems. The DualSPHysics code was employed in this study for investigation where physical data for validation was available from a full-scale model (Figure 3) of the system at the EPFL Laboratoire de Constructions Hydrauliques (LCH). In addition, some aspects of the on-site installation will be presented in this study as well.

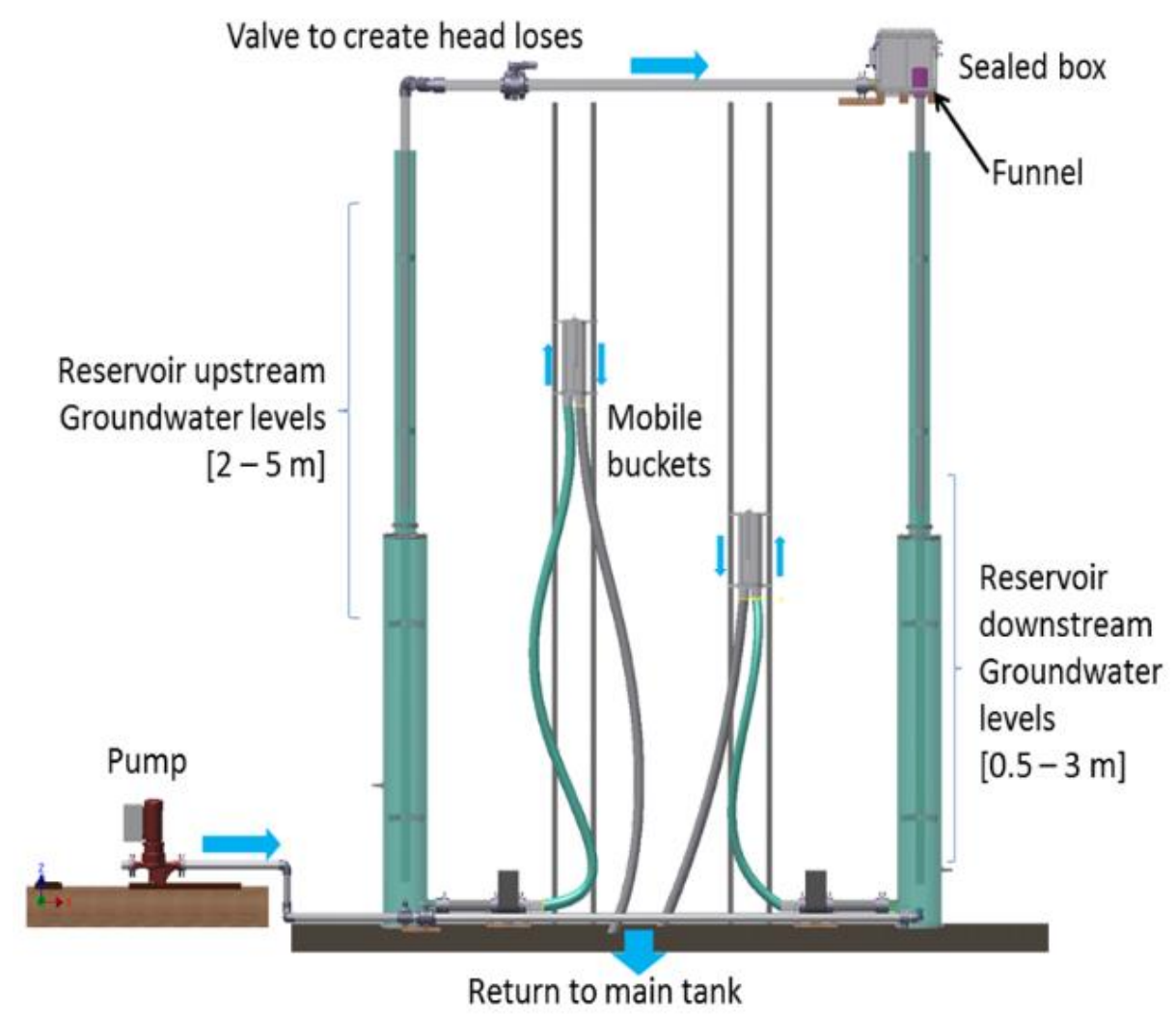

Figure 3: Schematic of physical model installed at the Laboratoire de Constructions Hydrauliques at the EPFL

\subsection{Physical Model}

The physical model installation consists of a self-priming siphon at the top of an inverse U-shape pipe as outlined in Figure 3. Two main tanks, with two mobile buckets, simulate the up- and down-stream reservoirs. Mobile buckets are used to mimic the varying water level, resp. pressure on each sides of the tunnel which represents the groundwater table in both reservoirs. The physical model was constructed at a scale of 1:1 to avoid scale effects from to the presence of air water mixture and due to and to the free-surface vortex vortices sensitivity to scaling [7] (viscosity and surface tension), particularly in a vacuum atmosphere. 
The key aspect of the system is the 'sealed box' which was designed using internal geometries to generate a free-surface vortex [7]. As it can be seen on the Figure 4, the red area lead the water into the funnel while creating a rotation of the fluid with an eccentric approach flow condition. The purpose of the vortex is to permit the entrainment and transport of air coming from leaks and other sources.

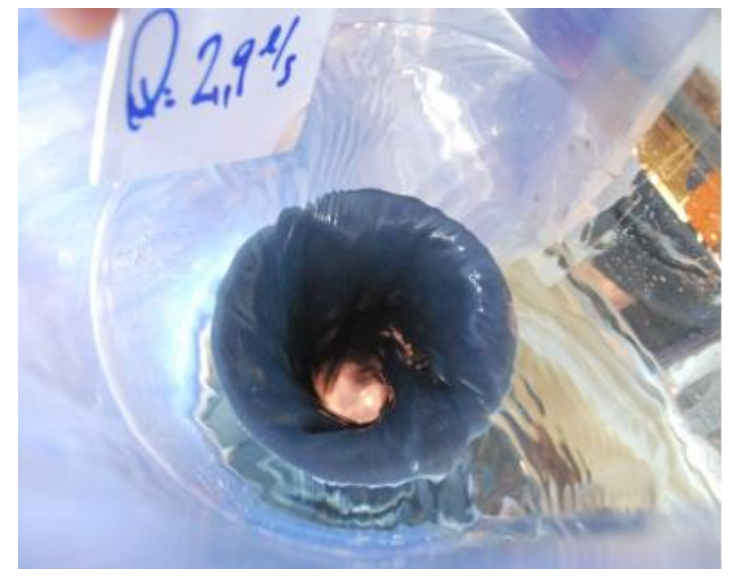

Figure 1: Presence of a vortex at a discharge of $2.9 \mathrm{l} / \mathrm{s}$

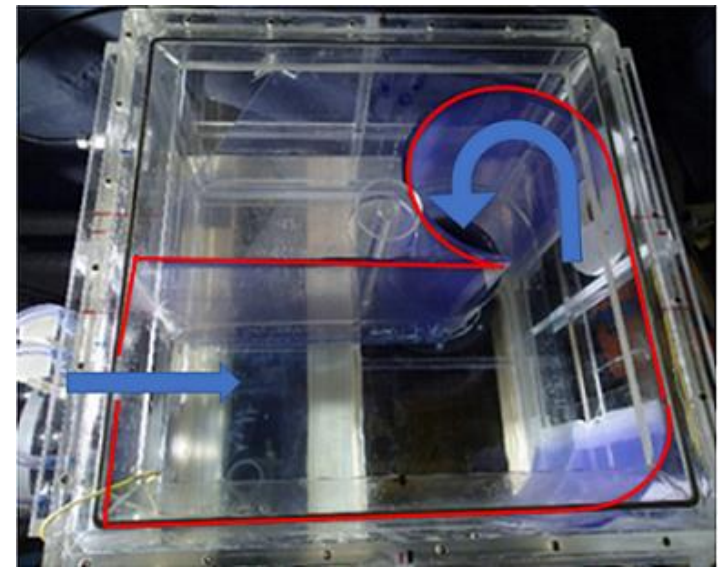

Figure 2: The vortex chamber

The water's height above the funnel (distance $h$ on Figure 5) has been measured for a range of discharges. The relation between the discharge $\mathrm{Q}_{2}$ and the water height $h$ is plotted in Figure 6. The behavior of the flow can be split into two distinct parts. By comparing the obtained results to existing hydraulic equations, Tulip (weir) spillway equation and vortex equation [7], the physical data agrees well with the equations in each flow regime.

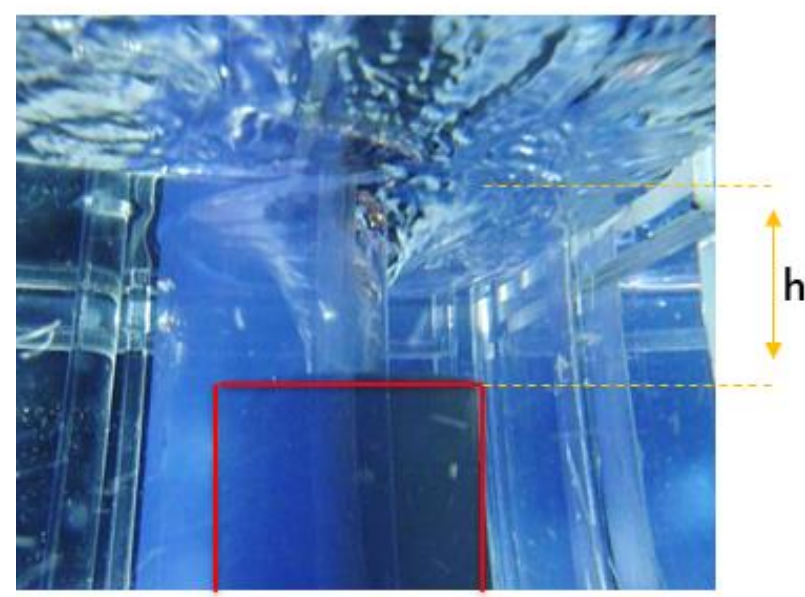

Figure 5: Water height above the funnel

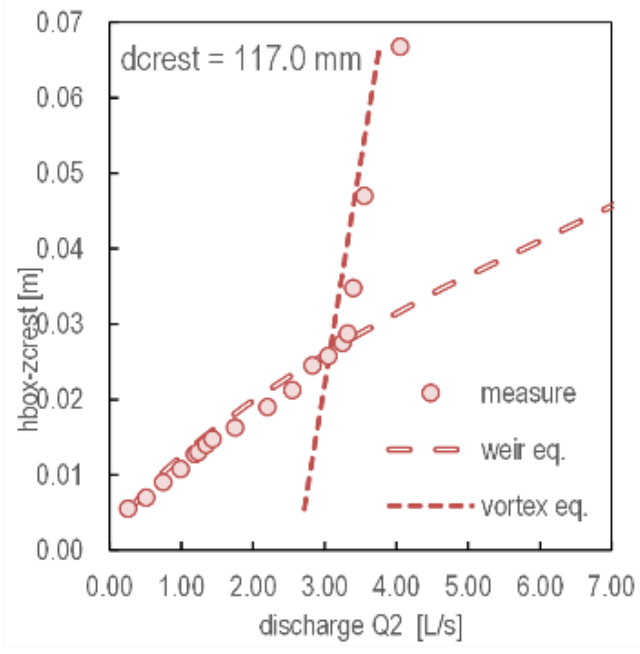

Figure 6: Results of the model experiment

The main conclusions of the experiment are the two distinct behaviors for low and high discharge. The purpose of this study is to simulate these conditions also with SPH and compare the results of the simulation with the Figure 6. Several Benchmark studies have been performed in free surface hydraulic flows e.g. flow over a weir [8] or very recently free surface jets impact [9]. As far as the authors are aware, this is the first application of SPH in simulating free-surface vortex flows. The reason of this investigation is to prove credibility of the SPH methodology by comparing the outcome to experimental results.

\section{SPH PRESENTATION}


SPH is a mesh-free Lagrangian simulation method initially developed to solve astrophysical problems in the 1970 's $[10,11,12]$. Nowadays, it can be applied to fluid dynamical problems and hydraulics applications such as hydraulic machines and hydraulic structures [13]. The fluid is modelled as multiple discrete particles used to represent the state and movement of the system [14]. Compared to a mesh-based simulation, SPH permits simpler determination of free-surfaces, boundaries, moving interfaces and complex geometry as it can be executed without a complex mesh. On the other hand, algorithms are not well-established and are usually computationally intensive.

\subsection{Mathematical model}

As the fluid in SPH consists of numerous solid particles, density $\rho$ is estimated using a weighted summation given by the following equation [12]:

$$
\rho(\boldsymbol{r})=\sum_{b} m_{b} W\left(\boldsymbol{r}-\boldsymbol{r}_{b}, h\right)
$$

where $W$ is a weight function called the smoothing kernel and has to be specified where the accuracy of density is derived, $h$ is a scale parameter determining the fall off rate of $W$. Each particle is represented by the index $b$, thus $m_{b}$ is the mass of each particle and $\boldsymbol{r}_{b}$ is its position in space.

Conservation laws of fluid dynamics are transformed to a suitable form using the kernel function. The Momentum equation is given by:

$$
\frac{d v}{d t}=-\frac{1}{\rho} \nabla P+\boldsymbol{g}+\boldsymbol{\Gamma}
$$

Where $\boldsymbol{g}$ is the gravitational acceleration and $\boldsymbol{\Gamma}$ refers to dissipative terms. In DualSPHysics, viscosity can be set using a predetermined, constant value given by the user or a value depending on the particle numbers used in the simulation. In this study, the viscosity has been fixed for quasi incompressible water to its kinematic viscosity, respectively $10^{-6} \mathrm{~m}^{2} / \mathrm{s}$ in order to decrease the needed amount of particles.

The continuity equation is then given by:

$$
\frac{d \rho_{a}}{d t}=\sum_{b} m_{b} \boldsymbol{v}_{a b} \cdot \nabla_{a} W_{a b}
$$

Where $\boldsymbol{v}_{a b}$ is the velocity difference between particle $a$ and $b$ and $W_{a b}$ is the chosen kernel function. Two different kernel functions can be chosen in DualSPHysics, the Cubic Spline or the Wendland function. For this study, Wendland is used by default as it offers greater stability and better convergence, it is also computationally more convenient [16].

\section{MODEL}

\subsection{Simulation set-up}

A 3D model of the vortex geometry was created initially using CATIA. For higher computational efficiency, the dimension of the chamber was reduced in the CAD model. Both length and width were reduced where only low flow velocities occur. This geometrical modification reduces consequently the number of particles but may add some inaccuracy in the results. The flow eccentricity leading to the vortex flow was maintained.

In order to avoid having a "dead zone" where particles can't move between the funnel and the chamber's wall, the location of the funnel has also been slightly moved away from the wall. This zone is due to the boundary layer present in DualSPHysics near solid walls. An excessively narrow gap between two walls can lead to a zone where fluid is trapped (see Figure 8) thus not representing true flow conditions realistically. The minimum gap is related to SPH smoothing kernel radius. The slight geometrical difference with respect to the real model geometry allows all over the domain a volume where fluid can flow. In mesh based RANS simulation, this could be solved by using much finer grid locally. 
A virtual head tank was added to the geometry in order to guarantee a certain realistic inflow boundary condition during the entire simulation. DualSPHysics has currently not implemented open boundary conditions (currently only periodic boundary conditions are available). As a specific inlet velocity is required at the vortex chamber's entrance, the chosen solution is a head tank with periodic boundary condition which takes the water from the outlet and puts it back at the top of the tank, similar to the experimental set-up with a closed hydraulic circuit. A total number of some 1.5 million particles is used. The same flow rate ( 0 to some $4 \mathrm{l} / \mathrm{s}$ ) was simulated as in the physical model. The corresponding head was extracted on a control section crossing the axis of the vortex core on the approach side (see Figure 8).

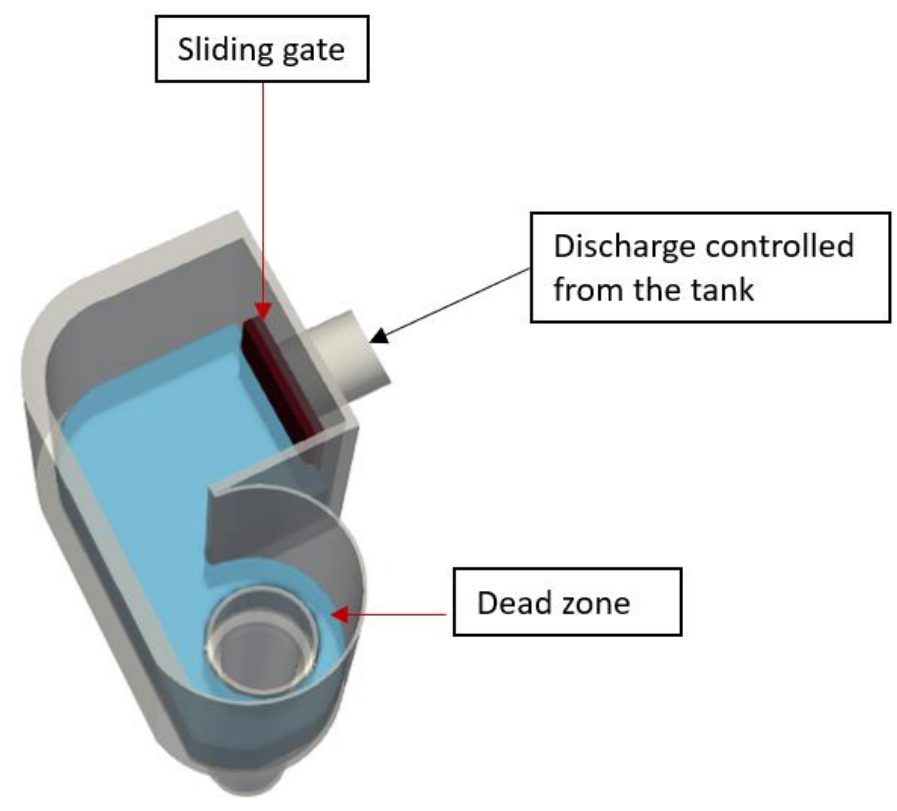

Figure 3: Model used for the simulation

To stabilize the flow in the chamber, a sliding gate has been added at the entrance of the vortex chamber. Multiple sequences of the gate's movement were tested in order to have a smooth increase of the discharge at the entrance. This smooth opening is needed in order to vary the height on top of the funnel with regards to the discharge. The smooth opening allows a quasi-steady flow in the chamber during each determination of the computed head above the funnel. Transient effects can thus be reduced similar to the physical experiments that required a certain duration to assure steady state conditions. By determining the water height, the simulation results can be compared with the experimental results.

\section{RESULTS AND DISCUSSION}

\subsection{Flow behavior}

Like the experiments made in the laboratory, the simulation has been performed for various discharges. During the simulation, the discharge increased gently until the end of the test. Transient behavior can consequently be prevented and the water height measurement will be more reliable. For the flow analysis, the simulation has been divided in three distinct parts:

1. The first part corresponded to the beginning of the simulation where the discharge was not high enough to generate a vortex. The discharge $Q$ ranges from 1 to approx. 2.5 1/s. In Figure 8, it is possible to observe the water discharging through the funnel radially where no rotation is present and gravity dominates the flow. Recirculation can be observed in the pre-chamber. The water that just entered the chamber hits the opposite wall and creates some counter flow towards the entrance. 


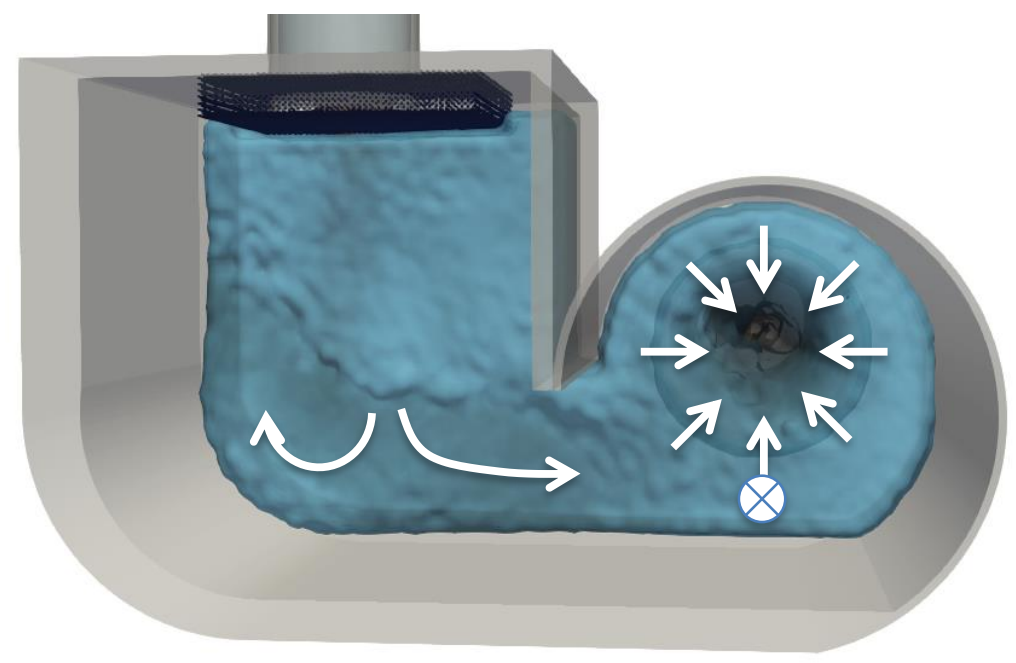

Figure 4: First part of the experiment, at low discharge there is no vortex, water leaves radially over the funnel crest, and the inflowing jet creates some counter flow towards the entrance. The head extraction position is given $\otimes$

2. The second part is near the middle of the simulation with a discharge $Q$ around 3 1/s. The vortex has been generated and its circulation increases as the discharge increases. A distinct vortex core is visible. The water that comes from the inlet chamber generates a pre-circulation due to the eccentricity of the geometry. A hydraulic jump can be observed in the chamber which characterizes the quick change of the water height in the chamber due to the opposite wall. The supply flow towards the funnel is more uniformly distributed over the flow width. A minor recirculation is also still present in the inlet channel. Slight water rotation can be observed around the funnel even though the incoming flow goes towards the funnel instead of around it [5].

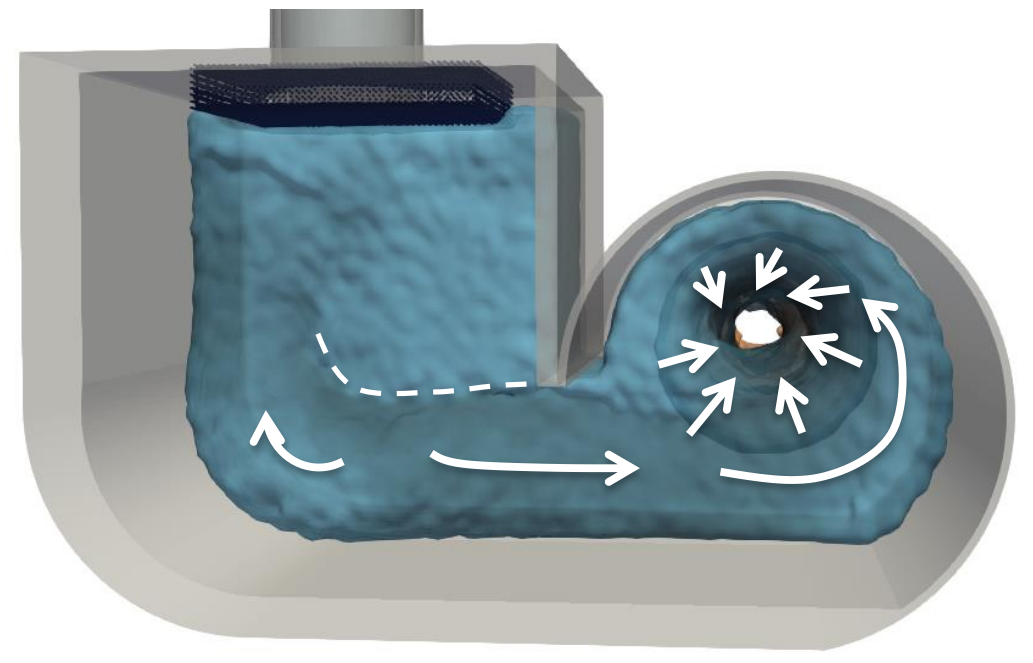

Figure 5: Second part of the experiment, the vortex is present, slight water rotation around the funnel occurs, the inflowing jet is weakened due to the jump in water level (----- line) which allows a more uniform flow towards the funnel, water still slightly recirculates in the inflow zone

3. The last part of the simulation concerns the vortex at its maximum strength. The discharge $Q$ ranges from approx. 3.5 to $4 \mathrm{l} / \mathrm{s}$. Due to the rotation of the water, the vortex also rotates on itself and takes a helicoid shape. Therefore, the vortex core cannot be seen from the top (see Figure 10). The same phenomena observed previously, a hydraulic jump and recirculation, are present. The low water height has increased its surface by going more in the direction of the entrance on the chamber's left side. 


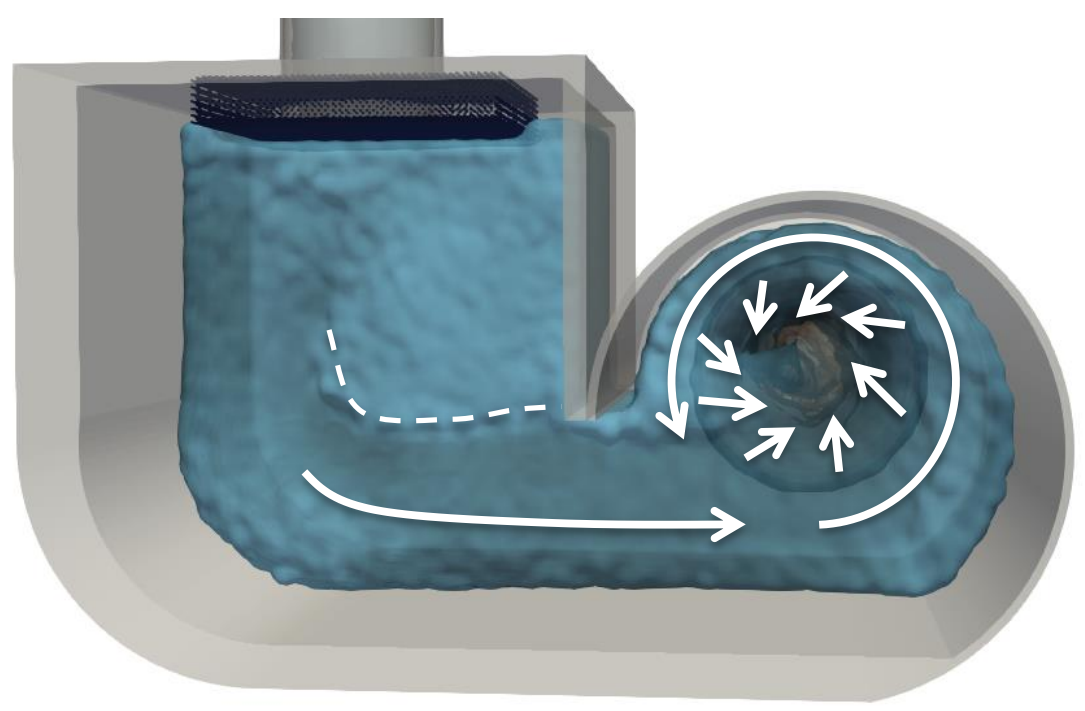

Figure 6: Generation of a strong free-surface vortex, there is a robust water rotation around the funnel, the inflowing jet is weakened due to the jump in water level (----- line) which allows a uniform flow towards the funnel

\subsection{Comparison with experimental}

A depth-discharge relationship was generated from the SPH simulation data. The weir and vortex equations have been used as in the previous base study [3] even with to some extent differing dimensions of the numerical set-up. The comparison with the experimentally validated equations shows a rather good fit between numerical and physical simulation, especially the transition phase for $Q=31 / \mathrm{s}$ is well reproduced which is the most related to the overall flow behavior. It is clearly visible that the simulation results follow two different gradients, separating weir flow and vortex flow as described previously. However, the results show that the simulated values diverge from the theoretical line especially for high discharges.

The effect of the adapted geometry to counteract the previously mentioned constraints of the SPH simulation cannot be fully disregarded, even if the weir and vortex equations seems applicable nevertheless. There is an inherent difficulty of computing and extracting flow discharge on the DualSPHysics solver and ParaView post processing. The values of $Q$, determined by multiplying the cross-section area with the mean particle velocities at the gate level, are assessed with more or less precision as particles are not necessarily present all over the control section because of boundary layer gaps, leading to an estimated error margin for the discharge in the range of $\pm 6 \%$. This error margin is mainly due to the occurrence of particles passing the control section area. A reduced distance between particles involving a much higher number of particles could reduce this inaccuracy. The exact determination of the head above the funnel is as well a delicate undertaking, as in the physical experiment. In the physical model, the head discharge tests have been performed with an open vortex chamber at atmospheric pressure, the water level being monitored with a needle gauche at the most calm position. In this simulation, the water level has been extracted in ParaView measuring the water surface elevation on a cross sectional plot at different times. Finally, due to the difficulty in imposing stationary boundary conditions and time constraints in running SPH, a simulation for each discharge has not been performed so far, this may introduce uncertainty due to transient effects. Their influence is difficult to quantify. 


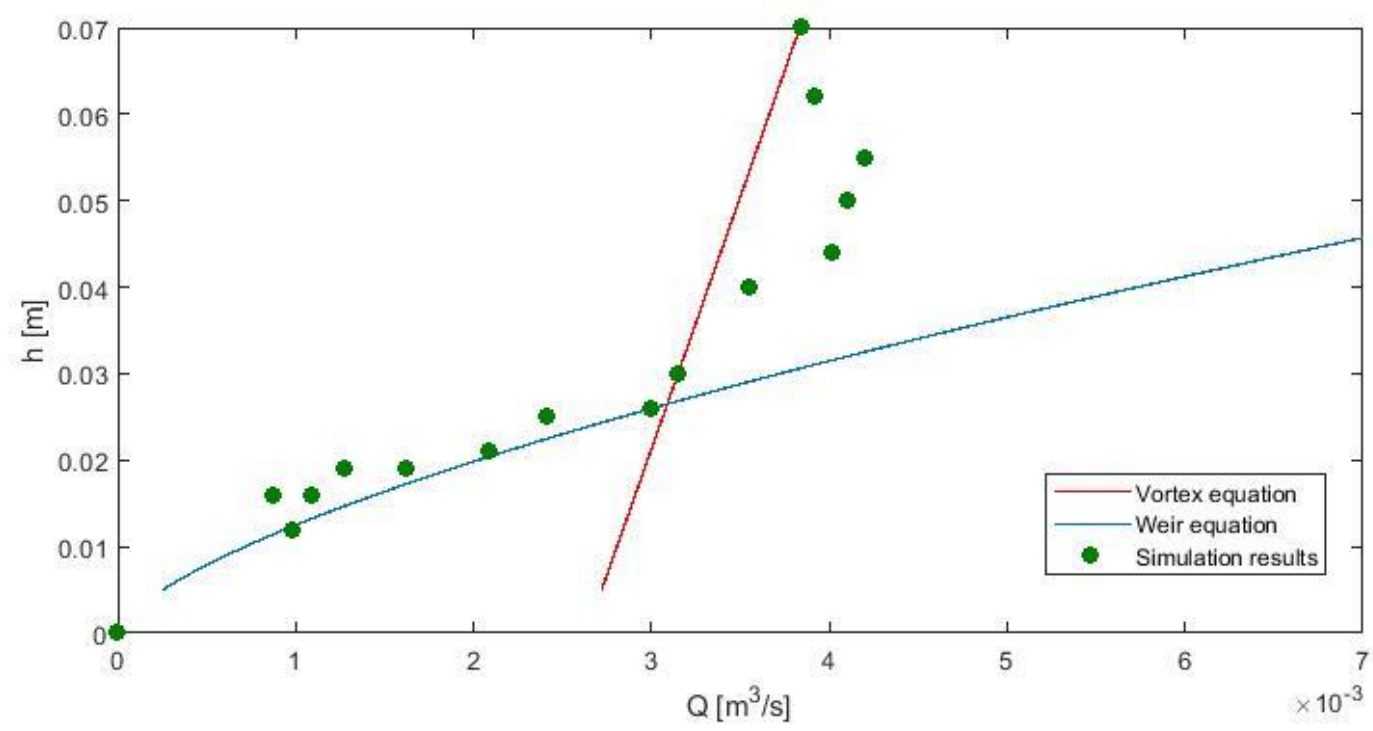

Figure 7: Results of the simulation

As far as the authors are aware, this is the first application of SPH simulations to a vortex chamber flow. Some uncertainty remains due to the various assumptions and approximations, difficulties with data extraction and definition of control sections as well as required adaptation of geometry compared to the physical set-up of the vortex chamber. At this stage, these results should be considered with caution and must be confirmed with new simulations awaiting SPH software improvements. However, based on the good qualitative agreement in the depth-discharge relationships and the free-surface characteristics, the study clearly exhibits and demonstrates the potential of the SPH tool in similar future studies.

\subsection{Further improvements}

With upgraded computational resources allowing models with higher number of particles, greater versatility in the SPH simulation of the vortex chamber could be achieved. It would also allow an implementation of simulations for the specific discharge values, leading to stationary flows thus removing uncertainty introduced from transient effects.

DualSPHysics is currently working on open inlet boundary conditions. In the future and already available in a $\beta$-version, the potentially erroneous head tank and sliding gate inlet boundary condition can be replaced by a better approximation of the real model through a flowrate assigned at the inlet.

In a next steep and already under way, flow simulation of the simpler circular vortex chamber with central exit hole on the bottom as studied physically and numerically with meshed RANS simulations in [6] is performed.

\section{CONCLUSIONS}

This project has shown some encouraging results in the application of SPH to vortex flows in a first time application. Despite the use of imperfect boundary conditions and discharge calculations, good qualitative agreements between flow behavior and depth-discharge relationships could be observed for both weir flow and vortex flow regimes. In particular, the transition could be simulated accurately. This actually shows that complex free-surface weir and vortex flows can be adequately simulated with DualSPHysics, despite its current limits and constraints. 


\section{ACKNOWLEDGEMENTS}

We would like to thank Prof. M. Sawley for giving us the opportunity to work on a subject that we choose. He also helped us to think about the right questions at the beginning of the project, focusing on the right tasks avoiding getting lost. The help provided to handle correctly the software was appreciated. We would also acknowledge the Swiss Federal Railways as the main stakeholder of the CEVA project in Geneva.

\section{REFERENCES AND CITATIONS}

1. Gabl, R, Gems, B., Plörer, M., Klar, R. Gschnitzer, T., Achleitner, S. and Aufleger, M. (2014). Numerical Simulations in Hydraulic Engineering. In: Hofstetter, G. (eds) Computational Engineering. Springer, pp 195-224, doi:10.1007/978-3-319-05933-4_8

2. Sawley, M. (2018). Particle-based methods, Master Course Book ME-476, EPFL, School of Engineering STI, Mechanical Engineering Section SGM

3. De Cesare, G., Essyad, K., Furlan, P., Khuong, V.N. and Mulligan, S. (2018). Experimental Study at Prototype Scale of a Self-priming Free-Surface Siphon, Advances in Hydroinformatics, Springer Water, pp. 899-912, doi:10.1007/978-981-10-7218-5_64

4. SBB (2019, March 8). Projet CEVA - Liaison ferroviaire Cornavin - Eaux-Vives - Annemasse, Retrieved from http://www.ceva.ch/

5. Mulligan, S., De Cesare, G., Casserly, J., and Sherlock, R. (2018). Understanding turbulent freesurface vortex flows using a Taylor-Couette flow analogy, Nature Scientific Reports, 824 (2018), doi:10.1038/s41598-017-16950-w

6. Mulligan, S. (2015). Experimental and numerical analysis of three-dimensional free-surface turbulent vortex flows with strong circulation. $\mathrm{PhD}$ dissertation.

7. Mulligan, S., Casserly, J. and Sherlock, R., (2016). Effects of geometry on strong free-surface vortices in subcritical approach flows. Journal of Hydraulic Engineering, 142(11), doi:10.1061/(ASCE)HY.1943-7900.0001194

8. Ferrari, A. (2010), SPH simulation of free surface flow over a sharp-crested weir, Advances in Water Resources, 33(3), doi:10.1016/j.advwatres.2009.12.005

9. Wu, J. Z. and Fang, L. (2018). Two-dimensional free-surface flow under gravity: A new benchmark case for SPH method, AIP Advances 8, 025220 (2018); doi:10.1063/1.5021678

10. Monaghan, J. J. (1992). Smoothed Particle Hydrodynamics, Annu. Rev. Astron. Astrophys., 30, $543-$ 574.

11. Monaghan, J. J. (1994). Simulating Free Surface Flows with SPH, J. Comput. Phys., 110(2), 399-406.

12. Monaghan, J. J. (2005), Smoothed particle hydrodynamics, Reports on Progress in Physics, 68(8), pp. 1703-1759, doi:10.1088/0034-4885/68/8/R01

13. Violeau, D. and Rogers, B. D. (2016). Smoothed particle hydrodynamics (SPH) for free-surface flows: past, present and future. Journal of Hydraulic Research, 54(1), pp.1-26.

14. Crespo, A. J. C., Domínguez, J. M., Gesteira, M. G., Barreiro, A., Rogers, B. D., Longshaw , S., Canelas, R., and Vacondio, R. (2016). User Guide for DualSPHysics code, University of Vigo, The University of Manchester and Johns Hopkins University 
15. Crespo, A. J. C., Dominguez, J. M., Rogers, B. D., Gomez-Gesteira, M., Longshaw, S., Canelas, R., Vacondio, R., Barreiro, A., and Garcia-Feal, O. (2015). DualSPHysics: Open-source parallel CFD solver based on Smoothed Particle Hydrodynamics (SPH), Comput. Phys. Comm., 187, 204-216

16. Dehnen, W., and Hossame, A. (2012). Improving convergence in smoothed particle hydrodynamics simulations without pairing instability, Mon. Not. R. Astron. S., 425, 1068-1082 